\title{
ADAPTIVE DESIGN OF CONTROLler AND SYNCHRONIZER FOR LU-XIAO CHAOTIC SYSTEM WITH UNKNOWN PARAMETERS
}

\author{
Sundarapandian Vaidyanathan ${ }^{1}$ \\ ${ }^{1}$ Research and Development Centre, Vel Tech Dr. RR \& Dr. SR Technical University \\ Avadi, Chennai-600 062, Tamil Nadu, INDIA \\ sundarvtu@gmail. com
}

\begin{abstract}
This paper establishes new results for the adaptive design of controller and synchronizer for the Lu-Xiao chaotic system (2012) with unknown parameters. First part of this paper involves the design of adaptive controller for the Lu-Xiao chaotic system to stabilize to its unstable equilibrium at the origin. The adaptive controller design is carried out using Lyapunov stability theory and adaptive control theory. The second part of this paper involves the design of adaptive synchronizer for identical Lu-Xiao chaotic systems with unknown parameters. The adaptive synchronizer design is carried out using Lyapunov stability theory and adaptive control theory. Numerical simulations using MATLAB have been shown to depict and validate the adaptive design of controller and synchronizer for the Lu-Xiao chaotic system with unknown parameters.
\end{abstract}

\section{KEYWORDS}

Adaptive Control, Adaptive Design, Adaptive Synchronization, Chaos, Lu-Xiao System.

\section{INTRODUCTION}

Since the observation of chaos phenomenon in weather models by Lorenz ([1], 1963), chaos theory has been received great attention in the nonlinear systems literature. Chaos theory finds applications in many areas in science and engineering such as physical systems [2], chemical systems [3], ecology [4], biology [5], secure communications [6-7], robotics [8], etc.

Control and synchronization of chaotic systems are important research problems with potential applications in many fields. By the control of a chaotic system, we mean the problem of finding a state feedback control law to stabilize a chaotic system around its unstable equilibrium [9-10].

By the synchronization of chaotic systems, we mean the problem of finding a control law attached to the slave system so as to synchronize the state trajectories of a pair of chaotic systems known as master and slave systems. In the last few decades, there has been a great interest for the synchronization of chaotic and hyperchaotic systems due to their applications.

There are many methods studied in the literature for chaos synchronization such as PC method [11], active control method [12-14], adaptive control method [15-16], time-delay feedback method [17], sampled-data feedback method [18], backstepping method [19-21], sliding mode control method [22-23], etc. 
International Journal of Computer Science \& Information Technology (IJCSIT) Vol 5, No 1, February 2013

In this paper, we derive new results for the adaptive design of stabilizing Lu-Xiao chaotic system ([24], 2012) to its unstable equilibrium at the origin. We also derive new results for the adaptive design of synchronizing identical Lu-Xiao chaotic systems with unknown parameters. Numerical simulations using MATLAB have been shown to illustrate our results for the Lu-Xiao chaotic system with unknown parameters.

\section{Analysis of Lu-Xiao ChaOtic System}

Lu-Xiao chaotic system ([24], 2012) is a novel chaotic system described by the dynamics

$$
\begin{aligned}
& \dot{x}_{1}=a\left(x_{2}-x_{1}\right)+x_{2} x_{3} \\
& \dot{x}_{2}=-b x_{1} x_{3}+c x_{1} \\
& \dot{x}_{3}=d x_{1} x_{2}-\varepsilon x_{3}
\end{aligned}
$$

where $x \in R^{3}$ is the state and $a, b, c, d, \varepsilon$ are constant, positive parameters of the system. $\mathrm{Lu}$ and Xiao observed chaotic behaviour in the system (1) when the parameter values are

$a=20, b=5, c=40, d=4$ and $\varepsilon=3$

The strange, double-scroll, chaotic attractor of the Lu-Xiao system is shown in Figure 1 .

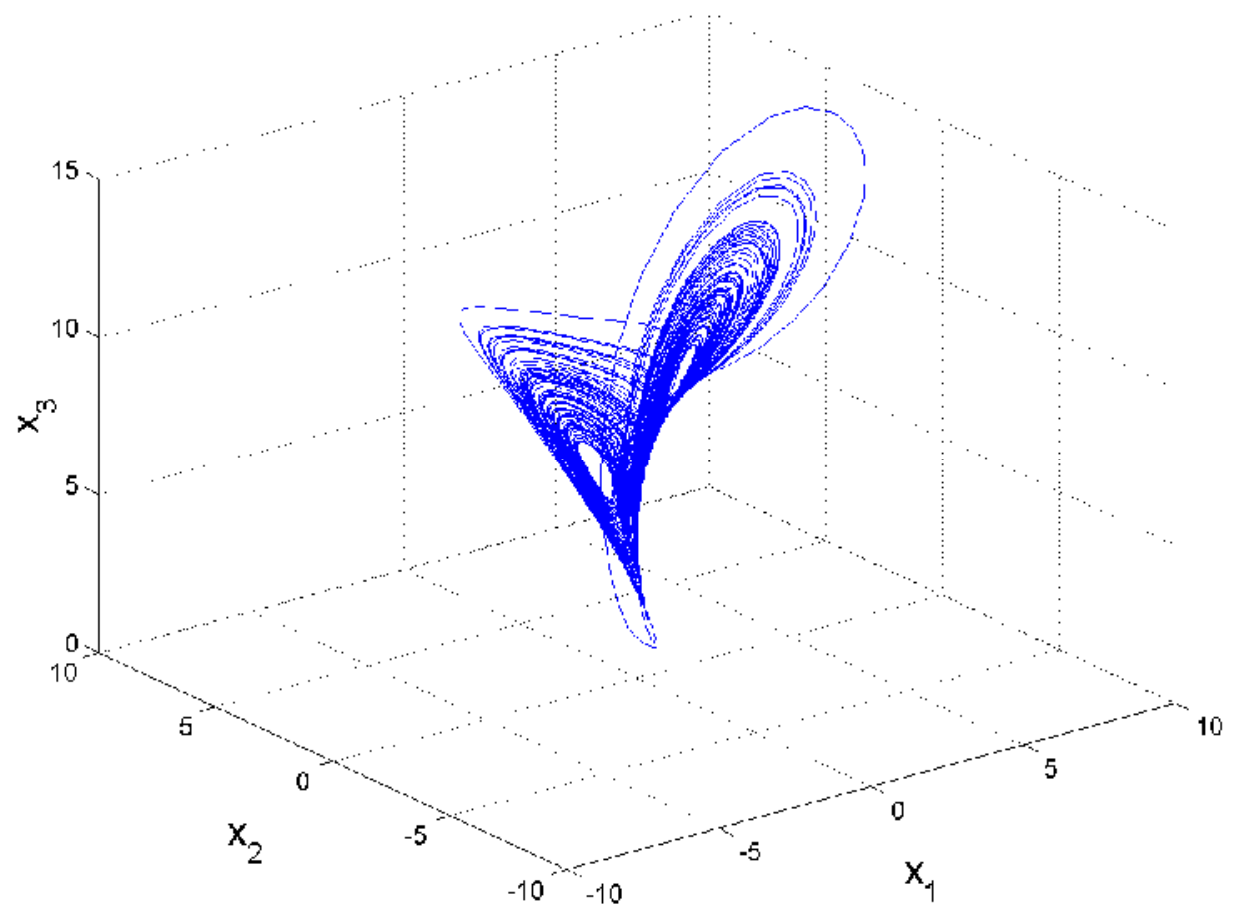

Figure 1. Strange Double-Scroll Attractor of the Lu-Xiao Chaotic System 
International Journal of Computer Science \& Information Technology (IJCSIT) Vol 5, No 1, February 2013

The linearization matrix of the Lu-Xiao system (1) at the equilibrium point $E_{0}=(0,0,0)$ is given by

$$
A=\left[\begin{array}{ccc}
-a & a & 0 \\
c & 0 & 0 \\
0 & 0 & -\varepsilon
\end{array}\right]
$$

which has the eigenvalues

$$
\lambda_{1}=-\varepsilon, \lambda_{2}=\frac{1}{2}\left(-a-\sqrt{a^{2}+4 a c}\right) \text { and } \lambda_{3}=\frac{1}{2}\left(-a+\sqrt{a^{2}+4 a c}\right) .
$$

Since $\lambda_{3}$ is a positive eigenvalues of $A$, it follows from Lyapunov's first stability theorem [25] that the Lu-Xiao chaotic system (1) is unstable at the equilibrium point $E_{0}=(0,0,0)$.

\section{Adaptive Control of the Lu-Xiao Chaotic System}

This section describes an adaptive design of a globally stabilizing feedback controller for the LuXiao chaotic system with unknown parameters. The design is carried out using adaptive control theory and Lyapunov stability theory.

We start the design procedure by considering a controlled Lu-Xiao chaotic system given by

$$
\begin{aligned}
& \dot{x}_{1}=a\left(x_{2}-x_{1}\right)+x_{2} x_{3}+u_{1} \\
& \dot{x}_{2}=-b x_{1} x_{3}+c x_{1}+u_{2} \\
& \dot{x}_{3}=d x_{1} x_{2}-\varepsilon x_{3}+u_{3}
\end{aligned}
$$

where $u_{1}, u_{2}, u_{3}$ are adaptive controllers to be found using the states $x_{1}, x_{2}, x_{3}$ and estimates $\alpha(t), \beta(t), \gamma(t), \delta(t), \eta(t)$ of the unknown parameters $a, b, c, d, \varepsilon$ of the system, respectively. Our design goal is to ensure that the controlled system (3) globally converges to the origin asymptotically for all values of the initial state $x(0) \in R^{3}$ and all initial values of the parameter estimates $\alpha(0), \beta(0), \gamma(0), \delta(0), \eta(0) \in R$.

For this purpose, we consider the adaptive controller given by

$$
\begin{aligned}
& u_{1}(t)=-\alpha(t)\left(x_{2}-x_{1}\right)-x_{2} x_{3}-k_{1} x_{1} \\
& u_{2}(t)=\beta(t) x_{1} x_{3}-\gamma(t) x_{1}-k_{2} x_{2} \\
& u_{3}(t)=-\delta(t) x_{1} x_{2}+\eta(t) x_{3}-k_{3} x_{3}
\end{aligned}
$$

where $k_{1}, k_{2}, k_{3}$ are positive gains.

Lu-Xiao dynamics (3), we get 
International Journal of Computer Science \& Information Technology (IJCSIT) Vol 5, No 1, February 2013

$$
\begin{aligned}
& \dot{x}_{1}=(a-\alpha)\left(x_{2}-x_{1}\right)-k_{1} x_{1} \\
& \dot{x}_{2}=-(b-\beta) x_{1} x_{3}+(c-\gamma) x_{1}-k_{2} x_{2} \\
& \dot{x}_{3}=(d-\delta) x_{1} x_{2}-(\varepsilon-\eta) x_{3}-k_{3} x_{3}
\end{aligned}
$$

We define the parameter estimation errors as

$$
\begin{aligned}
& e_{a}(t)=a-\alpha(t) \\
& e_{b}(t)=b-\beta(t) \\
& e_{c}(t)=c-\gamma(t) \\
& e_{d}(t)=d-\delta(t) \\
& e_{\varepsilon}(t)=\varepsilon-\eta(t)
\end{aligned}
$$

Using (6), the closed-loop dynamics (5) can be simplified to obtain the following:

$$
\begin{aligned}
& \dot{x}_{1}=e_{a}\left(x_{2}-x_{1}\right)-k_{1} x_{1} \\
& \dot{x}_{2}=-e_{b} x_{1} x_{3}+e_{c} x_{1}-k_{2} x_{2} \\
& \dot{x}_{3}=e_{d} x_{1} x_{2}-e_{\varepsilon} x_{3}-k_{3} x_{3}
\end{aligned}
$$

We adopt the Lyapunov approach for deriving an update law for the parameter estimates.

We consider the quadratic Lyapunov function given by

$$
V\left(x_{1}, x_{2}, x_{3}, e_{a}, e_{b}, e_{c}, e_{d}, e_{\varepsilon}\right)=\frac{1}{2}\left(x_{1}^{2}+x_{2}^{2}+x_{3}^{2}+e_{a}^{2}+e_{b}^{2}+e_{c}^{2}+e_{d}^{2}+e_{\varepsilon}^{2}\right) \text {, }
$$

which is a positive definite function on $R^{8}$.

A simple calculation from the equations (6) yields

$$
\begin{aligned}
& \dot{e}_{a}=-\dot{\alpha} \\
& \dot{e}_{b}=-\dot{\beta} \\
& \dot{e}_{c}=-\dot{\gamma} \\
& \dot{e}_{d}=-\dot{\delta} \\
& \dot{e}_{\varepsilon}=-\dot{\eta}
\end{aligned}
$$

By differentiating the Lyapunov function $V$ along the trajectories of (7) and using (9), we get

$$
\begin{aligned}
\dot{V}= & -k_{1} x_{1}^{2}-k_{2} x_{2}^{2}-k_{3} x_{3}^{2}+e_{a}\left[x_{1}\left(x_{2}-x_{1}\right)-\dot{\alpha}\right]+e_{b}\left[-x_{1} x_{2} x_{3}-\dot{\beta}\right] \\
& +e_{c}\left[x_{1} x_{2}-\dot{\gamma}\right]+e_{d}\left[x_{1} x_{2} x_{3}-\dot{\delta}\right]+e_{\varepsilon}\left[-x_{3}^{2}-\dot{\eta}\right]
\end{aligned}
$$

In view of the equation (10), the parameter estimates are updated by the following law: 
International Journal of Computer Science \& Information Technology (IJCSIT) Vol 5, No 1, February 2013

$$
\begin{aligned}
& \dot{\alpha}=x_{1}\left(x_{2}-x_{1}\right)+k_{4} e_{a} \\
& \dot{\beta}=-x_{1} x_{2} x_{3}+k_{6} e_{b} \\
& \dot{\gamma}=x_{1} x_{2}+k_{6} e_{c} \\
& \dot{\delta}=x_{1} x_{2} x_{3}+k_{7} e_{d} \\
& \dot{\eta}=-x_{3}^{2}+k_{8} e_{\varepsilon}
\end{aligned}
$$

where $k_{4}, k_{5}, k_{6}, k_{7}$ and $k_{8}$ are positive constants.

Next, we establish the following result for the adaptive control of Lu-Xiao chaotic system.

Theorem 1. The controlled Lu-Xiao chaotic system (3) having unknown system parameters $a, b, c, d, \varepsilon$ is globally and exponentially stabilized for all initial conditions $x(0) \in R^{3}$ and all initial values of the parameter estimates $\alpha(t), \beta(t), \gamma(t), \delta(t), \eta(t)$ for the unknown parameters $a, b, c, d, \varepsilon$, respectively, by the adaptive control law (4) and the parameter update law (11), where the gains $k_{i},(i=1, \ldots, 8)$ are positive constants. Also, the parameter estimation errors $e_{a}, e_{b}, e_{c}, e_{d}, e_{\varepsilon}$ converge to zero exponentially with time.

Proof. Substituting the parameter update law (11) into (10), we get

$$
\dot{V}=-k_{1} x_{1}^{2}-k_{2} x_{2}^{2}-k_{3} x_{3}^{2}-k_{4} x_{4}^{2}-k_{5} e_{a}^{2}-k_{6} e_{b}^{2}-k_{7} e_{c}^{2}-k_{8} e_{d}^{2}
$$

which is a negative definite function on $R^{8}$. By the direct method of Lyapunov [25], it follows that $x_{1}(t), x_{2}(t), x_{3}(t), e_{a}(t), e_{b}(t), e_{c}(t), e_{d}(t), e_{\varepsilon}(t)$ are globally exponentially stable.

\section{Numerical Results:}

For numerical simulations, we use the classical fourth-order Runge-Kutta method (MATLAB) with the step-size $h=10^{-8}$ to solve the Lu-Xiao system (3) with the adaptive control law (4) and the parameter update law (11). The parameters of the Lu-Xiao system (3) are taken as

$a=20, b=5, c=40, d=4$ and $\varepsilon=3$

For the adaptive and update laws, we take $k_{i}=5, \quad(i=1,2, \ldots, 8)$.

Suppose that the initial values of the parameter estimates are

$$
\alpha(0)=9, \quad \beta(0)=3, \quad \gamma(0)=4, \quad \delta(0)=6, \quad \eta(0)=8
$$

The initial state of the controlled Lu-Xiao system (3) is taken as

$$
x_{1}(0)=14, \quad x_{2}(0)=26, \quad x_{3}(0)=7
$$

The numerical simulations for the adaptive control of the Lu-Xiao chaotic system are depicted in Figures 2-5. 
International Journal of Computer Science \& Information Technology (IJCSIT) Vol 5, No 1, February 2013

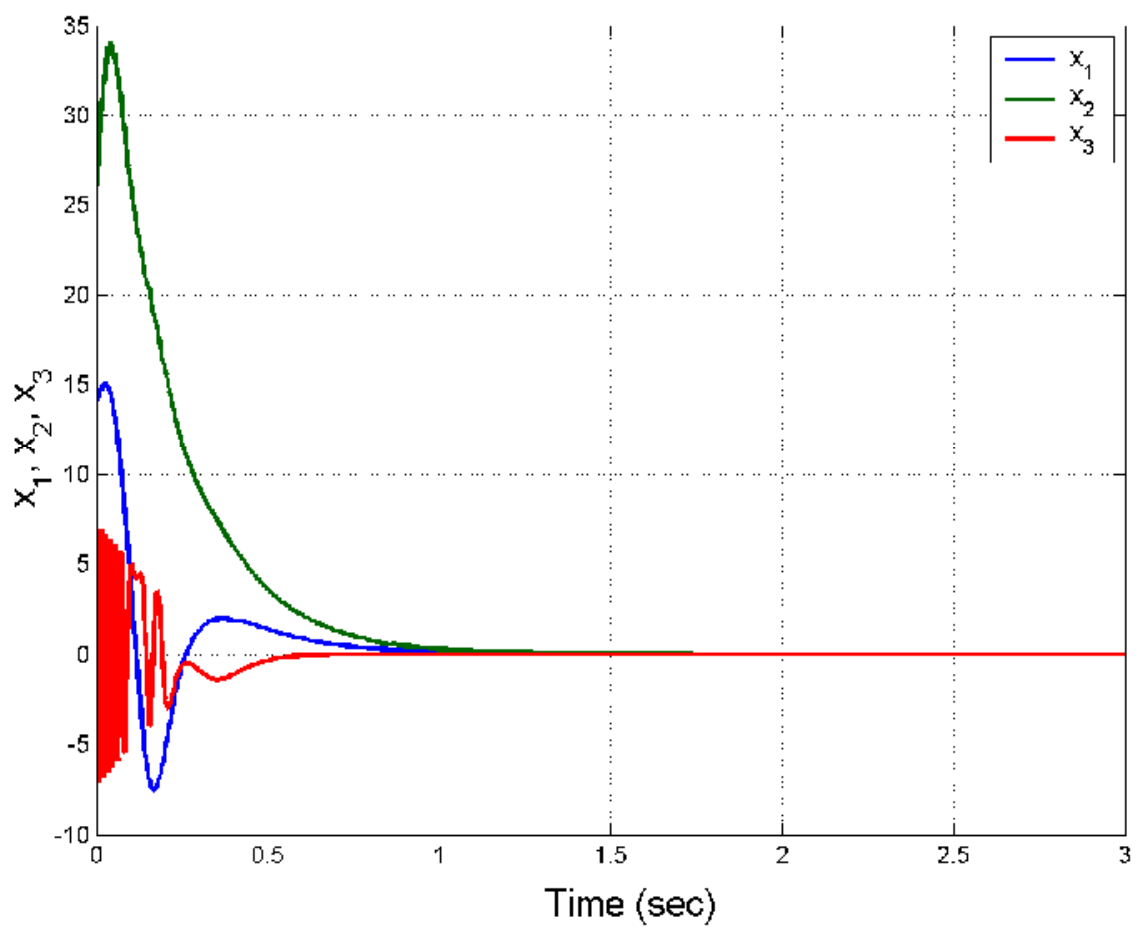

Figure 2. Time Responses of the Controlled Lu-Xiao System

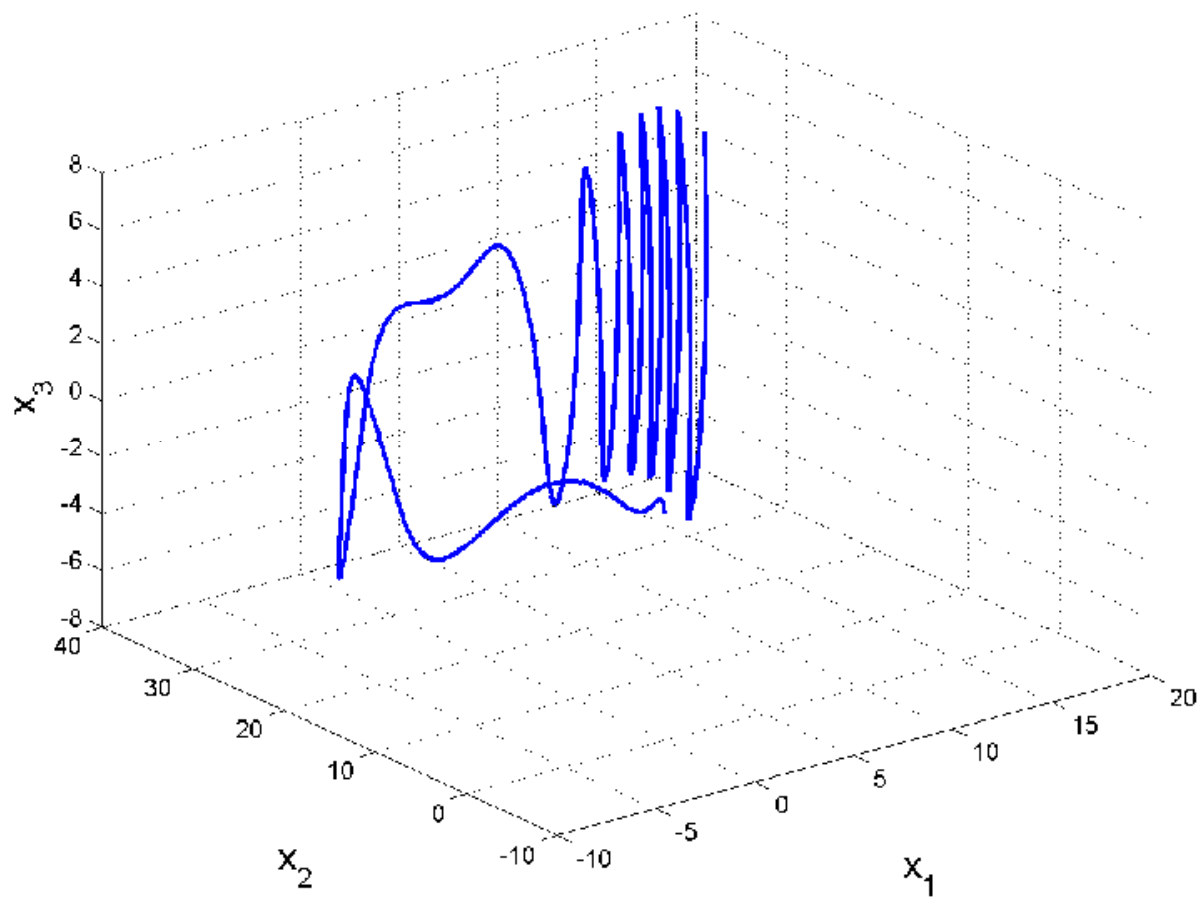

Figure 3. State Orbit of the Controlled Lu-Xiao System 
International Journal of Computer Science \& Information Technology (IJCSIT) Vol 5, No 1, February 2013

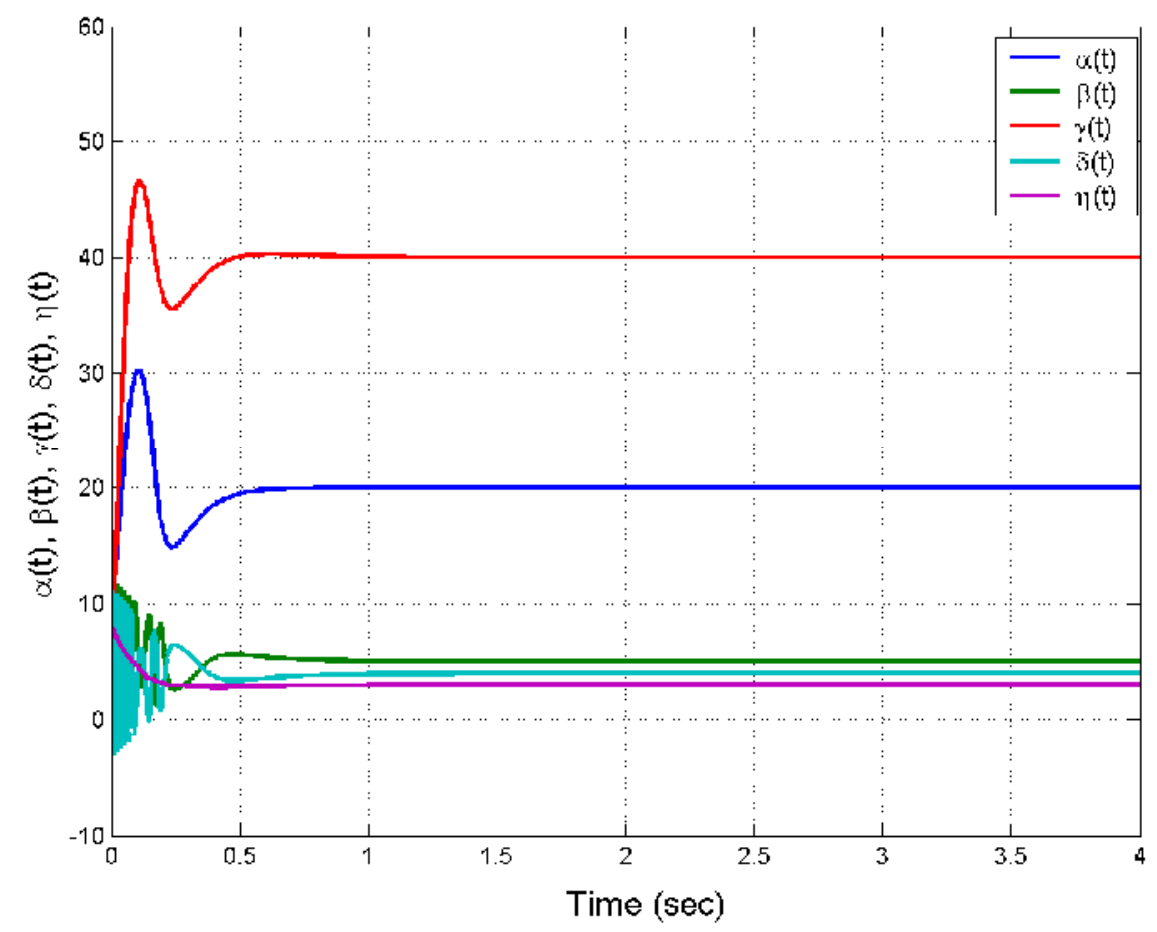

Figure 4. Time-History of the Parameter Estimates $\alpha(t), \beta(t), \gamma(t), \delta(t), \eta(t)$

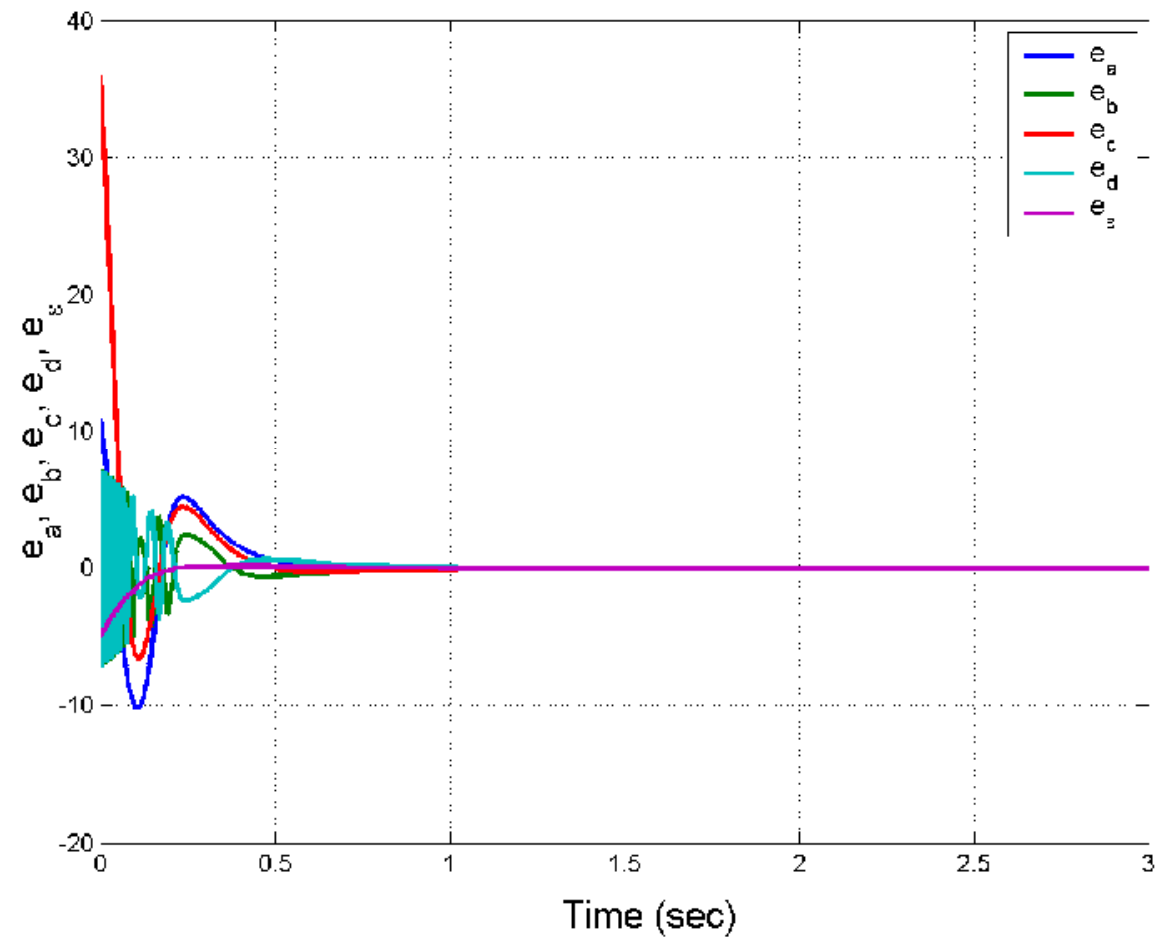

Figure 5. Time-History of the Parameter Estimation Errors $e_{a}(t), e_{b}(t), e_{c}(t), e_{d}(t), e_{\varepsilon}(t)$ 
International Journal of Computer Science \& Information Technology (IJCSIT) Vol 5, No 1, February 2013

\section{AdAPTIVE SYNCHRONIZATION OF IDENTICAL LU-XiAO SYSTEMS}

This section describes an adaptive design of global chaos synchronization of identical Lu-Xiao chaotic systems with unknown parameters. The design is carried out using adaptive control theory and Lyapunov stability theory.

As the master system, we take the Lu-Xiao dynamics described by

$$
\begin{aligned}
& \dot{x}_{1}=a\left(x_{2}-x_{1}\right)+x_{2} x_{3} \\
& \dot{x}_{2}=-b x_{1} x_{3}+c x_{1} \\
& \dot{x}_{3}=d x_{1} x_{2}-\varepsilon x_{3}
\end{aligned}
$$

where $x \in R^{3}$ is the state and $a, b, c, d$ are unknown system parameters.

As the slave system, we take the controlled Lu-Xiao dynamics described by

$$
\begin{aligned}
& \dot{y}_{1}=a\left(y_{2}-y_{1}\right)+y_{2} y_{3}+u_{1} \\
& \dot{y}_{2}=-b y_{1} y_{3}+c y_{1}+u_{2} \\
& \dot{y}_{3}=d y_{1} y_{2}-\varepsilon y_{3}+u_{3}
\end{aligned}
$$

where $y \in R^{3}$ is the state and $u_{1}, u_{2}, u_{3}$ are adaptive controllers to be found using the states $x_{1}, x_{2}, x_{3}$ and estimates $\alpha(t), \beta(t), \gamma(t), \delta(t), \eta(t)$ of the unknown parameters $a, b, c, d, \varepsilon$ of the system, respectively.

The synchronization error is defined by

$$
e_{i}=y_{i}-x_{i}, \quad(i=1,2,3)
$$

A simple calculation results in the error dynamics

$$
\begin{aligned}
& \dot{e}_{1}=a\left(e_{2}-e_{1}\right)+y_{2} y_{3}-x_{2} x_{3}+u_{1} \\
& \dot{e}_{2}=c e_{1}-b\left(y_{1} y_{3}-x_{1} x_{3}\right)+u_{2} \\
& \dot{e}_{3}=-\varepsilon e_{3}+d\left(y_{1} y_{2}-x_{1} x_{2}\right)+u_{3}
\end{aligned}
$$

Our design goal is to synchronize the Lu-Xiao chaotic systems (13) and (14) for all values of the initial state $x(0) \in R^{3}$ and all initial values of the parameter estimates. So, we take

$$
\begin{aligned}
& u_{1}(t)=-\alpha(t)\left(e_{2}-e_{1}\right)-y_{2} y_{3}+x_{2} x_{3}-k_{1} e_{1} \\
& u_{2}(t)=-\gamma(t) e_{1}+\beta(t)\left(y_{1} y_{3}-x_{1} x_{3}\right)-k_{2} e_{2} \\
& u_{3}(t)=\eta(t) e_{3}-\delta(t)\left(y_{1} y_{2}-x_{1} x_{2}\right)-k_{3} e_{3}
\end{aligned}
$$

where $\alpha, \beta, \gamma, \delta, \eta$ are estimates of the parameters $a, b, c, d, \varepsilon$ respectively, and $k_{1}, k_{2}, k_{3}$ are positive constants. 
International Journal of Computer Science \& Information Technology (IJCSIT) Vol 5, No 1, February 2013

By substituting the control law (17) into (16), we get the closed-loop error dynamics as

$$
\begin{aligned}
& \dot{e}_{1}=(a-\alpha)\left(e_{2}-e_{1}\right)-k_{1} e_{1} \\
& \dot{e}_{2}=(c-\gamma) e_{1}-(b-\beta)\left(y_{1} y_{3}-x_{1} x_{3}\right)-k_{2} e_{2} \\
& \dot{e}_{3}=-(\varepsilon-\eta) e_{3}+(d-\delta)\left(y_{1} y_{2}-x_{1} x_{2}\right)-k_{3} e_{3}
\end{aligned}
$$

We define parameter estimation errors as

$$
e_{a}=a-\alpha(t), e_{b}=b-\beta(t), e_{c}=c-\gamma(t), e_{d}=d-\delta(t), e_{\varepsilon}=\varepsilon-\eta(t)
$$

If we substitute (19) into (18), then the error dynamics simplifies to

$$
\begin{aligned}
& \dot{e}_{1}=e_{a}\left(e_{2}-e_{1}\right)-k_{1} e_{1} \\
& \dot{e}_{2}=e_{c} e_{1}-e_{b}\left(y_{1} y_{3}-x_{1} x_{3}\right)-k_{2} e_{2} \\
& \dot{e}_{3}=-e_{\varepsilon} e_{3}+e_{d}\left(y_{1} y_{2}-x_{1} x_{2}\right)-k_{3} e_{3}
\end{aligned}
$$

We adopt the Lyapunov approach for finding the update law for the estimates of the parameters.

We consider the quadratic Lyapunov function defined by

$V\left(e_{1}, e_{2}, e_{3}, e_{a}, e_{b}, e_{c}, e_{d}, e_{\varepsilon}\right)=\frac{1}{2}\left(e_{1}^{2}+e_{2}^{2}+e_{3}^{2}+e_{a}^{2}+e_{b}^{2}+e_{c}^{2}+e_{d}^{2}+e_{\varepsilon}^{2}\right)$,

which is a positive definite function on $R^{8}$.

A simple calculation from the equations (20) yields

$\dot{e}_{a}=-\dot{\alpha}, \quad \dot{e}_{b}=-\dot{\beta}, \quad \dot{e}_{c}=-\dot{\gamma}, \quad \dot{e}_{d}=-\dot{\delta}, \dot{e}_{\varepsilon}=-\dot{\eta}$

By differentiating $V$ along the trajectories of (22) and using (20), we get

$$
\begin{aligned}
\dot{V}= & -k_{1} x_{1}^{2}-k_{2} x_{2}^{2}-k_{3} x_{3}^{2}+e_{a}\left[e_{1}\left(e_{2}-e_{1}\right)-\dot{\alpha}\right]+e_{b}\left[-e_{2}\left(y_{1} y_{3}-x_{2} x_{3}\right)-\dot{\beta}\right] \\
& +e_{c}\left[e_{1} e_{2}-\dot{\gamma}\right]+e_{d}\left[e_{3}\left(y_{1} y_{2}-x_{1} x_{2}\right)-\dot{\delta}\right]+e_{\varepsilon}\left[-e_{3}^{2}-\dot{\eta}\right]
\end{aligned}
$$

In view of equation (23), the parameter estimates are updated by the following law:

$$
\begin{aligned}
& \dot{\alpha}=e_{1}\left(e_{2}-e_{1}\right)+k_{4} e_{a} \\
& \dot{\beta}=-e_{2}\left(y_{1} y_{3}-x_{1} x_{3}\right)+k_{6} e_{b} \\
& \dot{\gamma}=e_{1} e_{2}+k_{6} e_{c} \\
& \dot{\delta}=e_{3}\left(y_{1} y_{2}-x_{1} x_{2}\right)+k_{7} e_{d} \\
& \dot{\eta}=-e_{3}^{2}+k_{8} e_{\varepsilon}
\end{aligned}
$$

where $k_{4}, k_{5}, k_{6}, k_{7}, k_{8}$ are positive constants. 
International Journal of Computer Science \& Information Technology (IJCSIT) Vol 5, No 1, February 2013

Theorem 2. The identical Lu-Xiao systems (13) and (14) with unknown parameters $a, b, c, d, \varepsilon$ are globally and exponentially stabilized for all initial conditions $x(0), y(0) \in R^{3}$ and all initial values of the parameter estimates $\alpha(t), \beta(t), \gamma(t), \delta(t), \eta(t)$ for the unknown parameters $a, b, c, d, \varepsilon$, respectively, by the adaptive control law (17) and the parameter update law (24), where the gains $k_{i},(i=1, \ldots, 8)$ are positive constants. Also, the parameter estimation errors $e_{a}, e_{b}, e_{c}, e_{d}, e_{\varepsilon}$ converge to zero exponentially with time.

Proof. Substituting (24) into (23), we get

$$
\dot{V}=-k_{1} e_{1}^{2}-k_{2} e_{2}^{2}-k_{3} e_{3}^{2}-k_{4} e_{4}^{2}-k_{5} e_{a}^{2}-k_{6} e_{b}^{2}-k_{7} e_{c}^{2}-k_{8} e_{d}^{2}
$$

From (25), we find that $\dot{V}$ is a negative definite function on $R^{8}$.

Thus, by Lyapunov stability theory [25], it is immediate that the synchronization error and the parameter error decay to zero exponentially with time for all initial conditions.

\section{Numerical Results:}

For numerical simulations, we use the classical fourth-order Runge-Kutta method (MATLAB) with the step-size $h=10^{-8}$ to solve the Lu-Xiao systems (13) and (14) with the adaptive control law (17) and the parameter update law (24).

The parameters of the Lu-Xiao system are taken as

$$
a=20, b=5, c=40, d=4 \text { and } \varepsilon=3
$$

For the adaptive and update laws, we take $k_{i}=5, \quad(i=1,2, \ldots, 8)$.

Suppose that the initial values of the parameter estimates are

$$
\alpha(0)=12, \quad \beta(0)=20, \quad \gamma(0)=4, \quad \delta(0)=16, \quad \eta(0)=5
$$

Suppose that the initial values of the master system (13) are

$$
x_{1}(0)=7, \quad x_{2}(0)=-5, \quad x_{3}(0)=26
$$

Suppose that the initial values of the slave system (14) are

$$
y_{1}(0)=-24, \quad y_{2}(0)=18, \quad y_{3}(0)=5
$$

Figure 6 shows the adaptive chaos synchronization of the identical Lu-Xiao systems.

Figure 7 shows the time-history of the synchronization error $e_{1}, e_{2}, e_{3}$.

Figure 8 shows the time-history of the parameter estimates $\alpha(t), \beta(t), \gamma(t), \delta(t), \eta(t)$.

Figure 9 shows the time-history of the parameter estimation errors $e_{a}, e_{b}, e_{c}, e_{d}, e_{\varepsilon}$. 
International Journal of Computer Science \& Information Technology (IJCSIT) Vol 5, No 1, February 2013
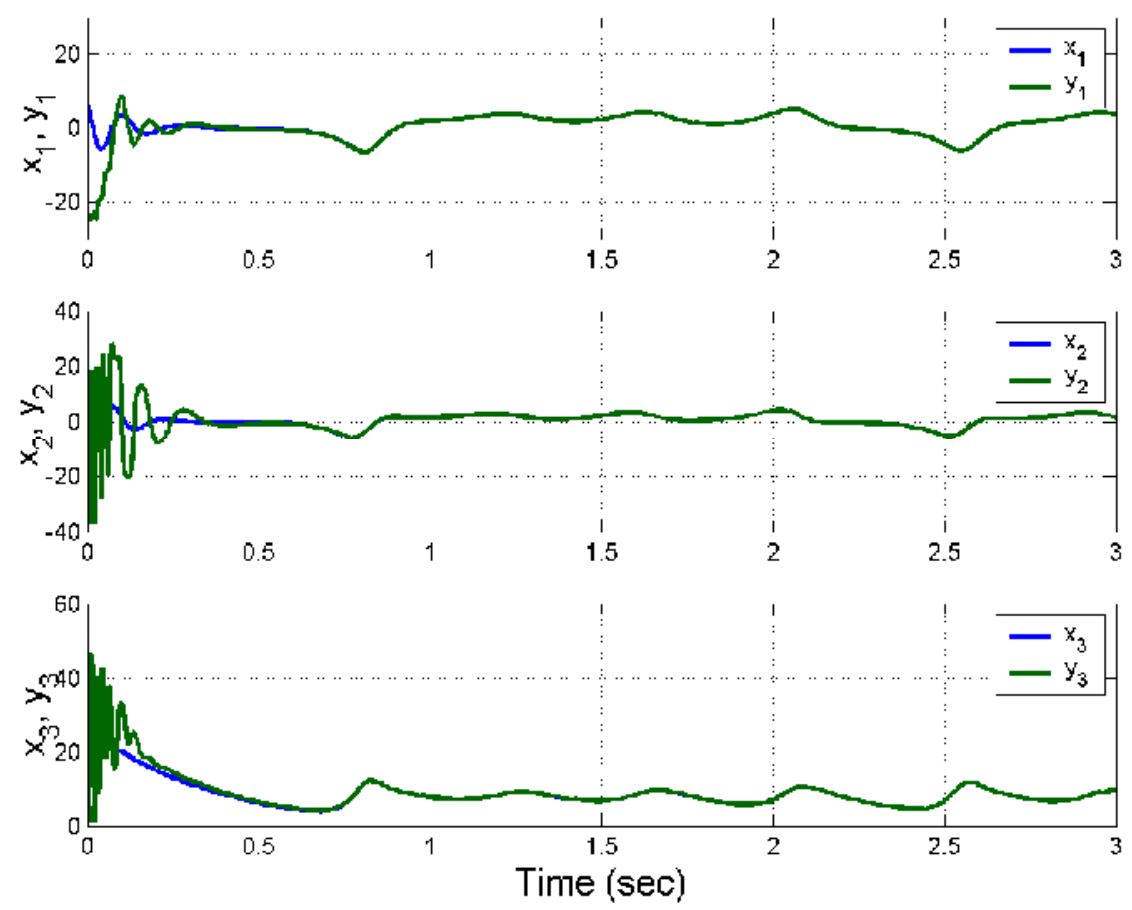

Figure 6. Adaptive Synchronization of the Lu-Xiao Systems

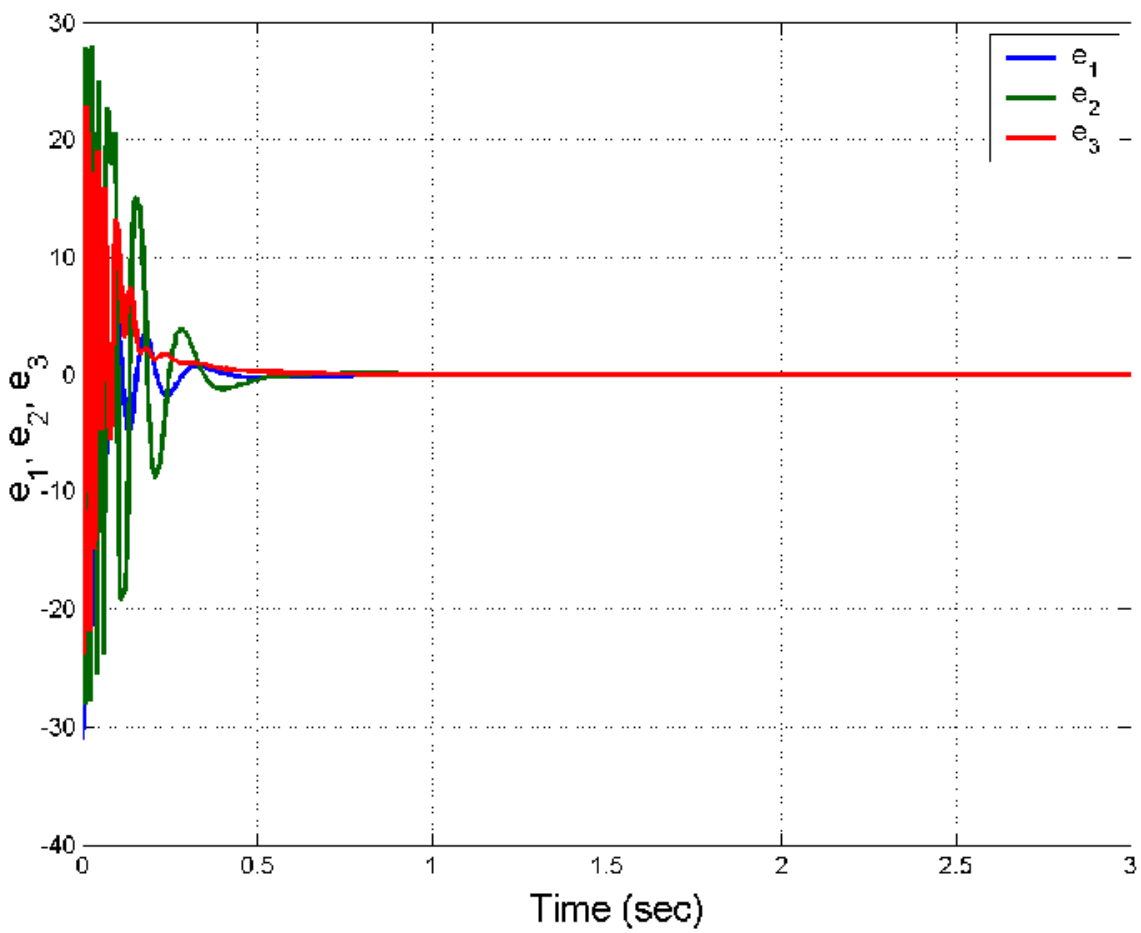

Figure 7. Time-History of the Synchronization Errors $e_{1}, e_{2}, e_{3}$ 
International Journal of Computer Science \& Information Technology (IJCSIT) Vol 5, No 1, February 2013

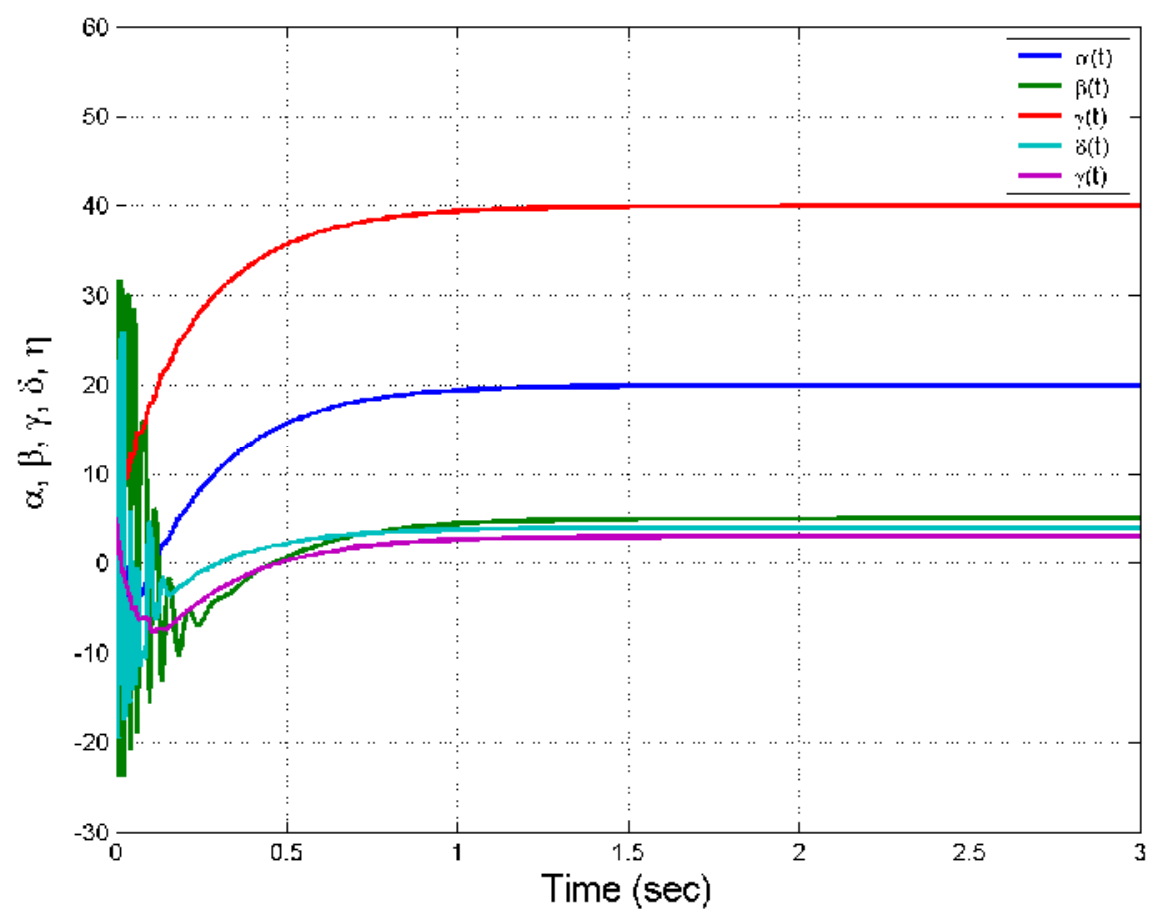

Figure 8. Time-History of the Parameter Estimates $\alpha(t), \beta(t), \gamma(t), \delta(t), \eta(t)$

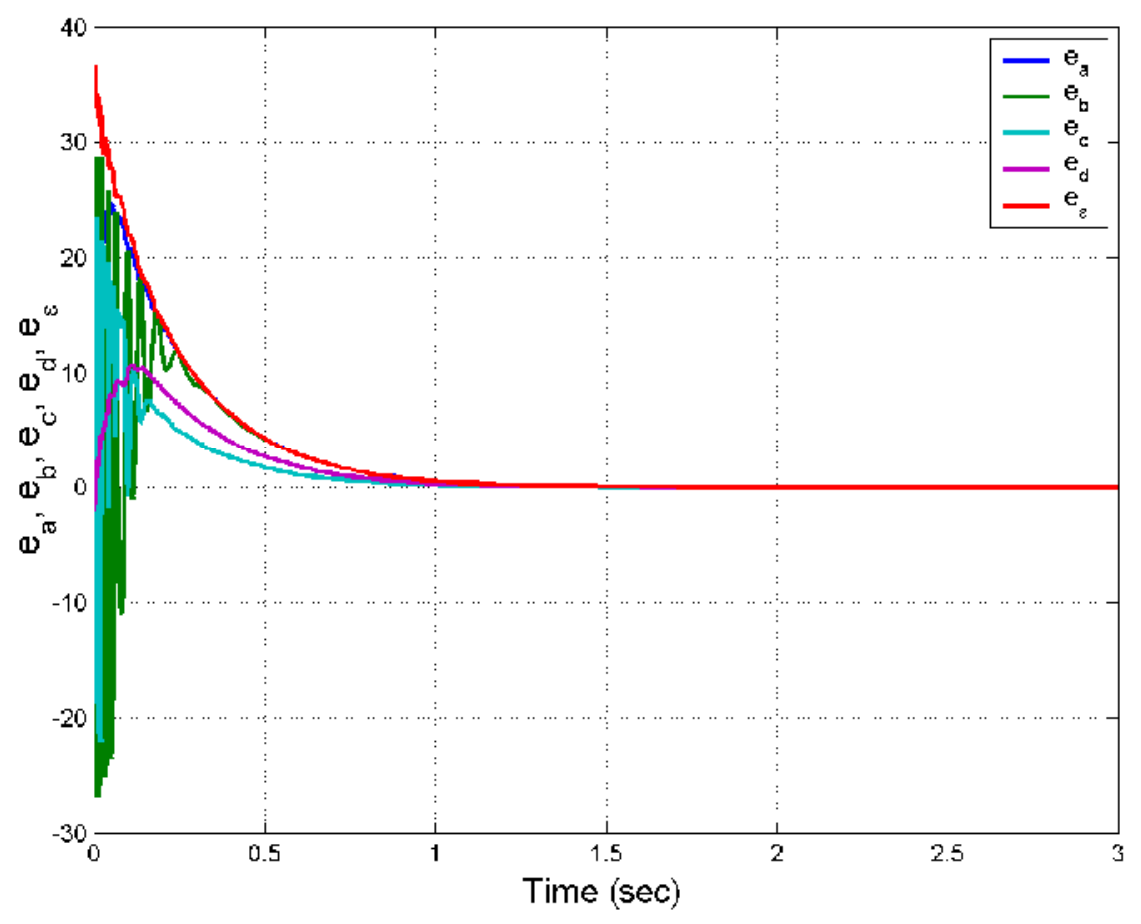

Figure 9. Time-History of the Parameter Estimation Errors $e_{a}, e_{b}, e_{c}, e_{d}, e_{\varepsilon}$ 
International Journal of Computer Science \& Information Technology (IJCSIT) Vol 5, No 1, February 2013

\section{Conclusions}

In this paper, we found new results for the adaptive design of controller and synchronizer for the Lu-Xiao chaotic system (2012) with unknown system parameters. The main theorems of this paper have been proved via adaptive control theory and Lyapunov stability theory. Numerical simulations using MATLAB were shown to depict and demonstrate the proposed adaptive control and synchronization schemes for the Lu-Xiao chaotic system.

\section{REFERENCES}

[1] Lorenz, E.N. (1963) "Deterministic nonperiodic flow," J. Atmos. Sci., Vol. 20, pp 130-141.

[2] El Naschie, M.S. (1996) "Introduction to chaos, information and diffusion in quantum physics," Chaos, Solitons \& Fractals, Vol. 7, No. 5, pp 6-8.

[3] Doherty, M.F. \& Ottino, J.M. (1988) "Chaos in deterministic systems: Strange attractors, turbulence and applications in chemical engineering," Chemical Engineering Science, Vol. 43, No. 2, pp 139183.

[4] Upadhyay, R.K. (2009) "Dynamics of an ecological model living on the edge of chaos," Applied Math. Computation, Vol. 210, No. 2, pp 455-464.

[5] Gakkhar, S. \& Naji, R. (2003) "Existence of chaos in two-prey, one predator system," Chaos, Solitons \& Fractals, Vol. 17, pp 639-649.

[6] Zhang, B., Chen, M. \& Zhou, D. (2006) "Chaotic secure communication based on particle filtering," Chaos, Solitons \& Fractals, Vol. 30, No. 5, pp 1273-1280.

[7] Wang, X., Zhang, W., Guo, W. \& Zhang, J. (2013) "Secure chaotic system with application to chaotic ciphers," Information Sciences, Vol. 221, No. 1, pp 555-557.

[8] Nakamura, Y. \& Sekiguchi, A. (2001) "Chaotic mobile robot”, IEEE Trans. Robotics \& Automation, Vol. 17, pp 898-904.

[9] Ott, E., Grebogi, C. \& Yorke, J.A. (1990) "Controlling chaos", Physical Review Letters, Vol. 64, pp 1196-1199.

[10] Wang, X., Tian, L. \& Yu, L. (2006) "Adaptive control and slow manifold analysis of a new chaotic system," International Journal of Nonlinear Science, Vol. 21, pp 43-49.

[11] Pecora, L.M. \& Carroll, T.L. (1990) "Synchronization in chaotic systems", Phys. Rev. Lett., Vol. 64, pp 821-824.

[12] Tang, R.A., Liu, Y.L. \& Xue, J.K. (2009) "An extended active control for chaos synchronization," Phy. Lett. A, Vol. 373, No. 16, pp 1449-1454.

[13] Chen, H.H. Sheu, G.J., Lin, Y.L. \& Chen, C.S. (2009) "Chaos synchronization between two different chaotic systems via nonlinear feedback control,” Nonlinear Analysis, Vol. 70, No. 12, pp 4393-4401.

[14] Sundarapandian, V. (2011) "Global chaos synchronization of Lorenz and Pehlivan chaotic systems by nonlinear control”, International Journal of Advances in Science and Technology, Vol. 2, No. 3, pp 19-28.

[15] Zhang, Z., Wang, Y. \& Du, Z. (2012) "Adaptive synchronization of single-degree-of-freedom oscillators with unknown parameters," Applied Mathematics \& Computation, Vol. 218, No. 12, pp 6833-6840.

[16] Sundarapandian, V. (2011) "Adaptive control and synchronization of hyperchaotic Cai system", International Journal of Control Theory \& Computer Modeling, Vol. 1, No. 1, pp 1-13.

[17] Ghosh, D., Saha, P. \& Chowdhury, A.R. (2007) "On synchronization of a forced delay dynamical system via the Galerkin approximation,” Comm. Nonlinear Sci. Num. Sim., Vol. 12, No. 6, pp 928 941.

[18] Gan, Q. \& Liang, Y. (2012) "Synchronization of chaotic neural networks with time delay in the leakage term and parametric uncertainties based on sampled-data control," J. Franklin Institute, Vol. 349, No. 6, pp 1955-1971.

[19] Li, G.H. (2006) "Projective synchronization of chaotic system using backstepping control," Chaos, Solitons \& Fractals, Vol. 29, No. 2, pp 490-494.

[20] Yu, Y.G. \& Zhang, S.C. (2006) "Adaptive backstepping synchronization of uncertain chaotic systems", Chaos, Solitons and Fractals, Vol. 27, pp 1369-1375. 
International Journal of Computer Science \& Information Technology (IJCSIT) Vol 5, No 1, February 2013

[21] Suresh, R. \& Sundarapandian, V. (2012) "Global chaos synchronization of Windmi and Coullet chaotic systems by backstepping control”, Far East Journal of Mathematical Sciences, Vol. 67, No. 2, pp 265-282.

[22] Yau, H.T. (2004) "Design of adaptive sliding mode controller for chaos synchronization with uncertainties," Chaos, Solitons \& Fractals, Vol. 22, No. 2, pp 341-347.

[23] Sundarapandian, V. (2011) "Global chaos synchronization of four-wing chaotic systems by sliding mode control”, International Journal of Control Theory \& Computer Modeling, Vol., 1 No. 1, pp 1531.

[24] Lu, H. \& Xiao, X. (2012) “Analysis of a novel autonomous chaotic system," International Journal of Advancements in Computing Technology, Vol. 4, No. 1, pp 248-255.

[25] Hahn, W. (1967) The Stability of Motion, Springer, New York.

\section{AUTHOR}

Dr. V. Sundarapandian earned his D.Sc. degree in Electrical and Systems Engineering from Washington University, St. Louis, USA in May 1996. He is a Professor and Dean at the Research and Development Centre, Vel Tech Dr. RR \& Dr. SR Technical University, Chennai, Tamil Nadu, India. He has published over 300 refereed papers in international journals. He has published over 180 papers in National and International Conferences. He is the Editor-in-Chief of the AIRCC Journals - International Journal of Instrumentation and Control Systems, International Journal of Control Systems and Computer Modelling, International Journal of Information Technology, Control and Automation, International Journal of Chaos, Control, Modelling and Simulation, and

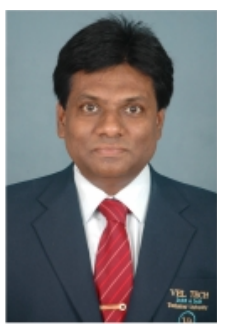
International Journal of Information Technology, Modeling and Computing. His research interests are Control Systems, Chaos Theory, Intelligent Computing, Optimal Control, Operations Research, Mathematical Modelling and Computational Science. He has delivered several Key Note Addresses in Control Systems, Chaos and Computational Science in International Conferences. 